\title{
Ophthalmologisches bei Conrad Geßner (1516-1565)
}

\author{
Von M. H. Koelbing ${ }^{1}$
}

Conrad Geßner gehört zu den Gelehrten, die in engen Verhältnissen Enormes geleistet haben. Er war, wie DIEPGEN ${ }^{2}$ sagt, «einer der universellsten Gelehrten, die die Wissenschaftsgeschichte kennt». In Basel und Montpellier hatte er Medizin studiert - mit Unterbrüchen, welche die pekuniäre Not diktierte. 1541 promovierte ihn die Medizinische Fakultät zu Basel zum Doktor der Medizin ${ }^{3}$. Es war wohl weniger der Drang zum Heilen und Helfen, der Geßner Arzt werden ließ, als vielmehr die Tatsache, daß von allen Universitätsdisziplinen einzig die Medizin sich mit dem Studium der Natur befaßte. Die Anatomie, zu seiner Studienzeit noch vor-vesalianisch, ganz nach GALEN gelehrt, hat Geßner offenbar nicht fasziniert. Um so intensiver beschäftigte er sich während seines ganzen Lebens mit Pharmazie und Botanik. In seinem unablässigen Bestreben, die Pflanzenwelt - aber auch Tiere und Mineralien - möglichst vollständig kennen zu lernen und zu beschreiben, ließ er freilich den Bereich des Pharmazeutisch-Nützlichen weit hinter sich zurück; die Erforschung der Natur wurde ihm zur eigentlichen Lebensaufgabe. MrLT ${ }^{4}$ hat dazu geschrieben: «Was Geßner zu einem Begründer moderner, abendländischer Naturwissenschaft macht, sind nicht besondere Entdeckungen, nicht erkenntnistheoretische Spekulationen und Systeme, sondern ein neuartiges, wirklichkeitsnahes Sehen. Hatte man sich vor ihm vornehmlich um die wissenschaftliche Literatur über die Natur gekümmert, hat er als einer der ersten die Geschöpfe der Natur aus eigener Anschauung möglichst genau in ihren äußern Erscheinungsformen in Wort und Bild wiedergegeben und damit den Grund zu ihrer Inventarisation gelegt.» Dasselbe neue, wirklichkeitsnahe Sehen hat Andreas Vesal zum Begründer der modernen Anatomie werden lassen; es hat das ärztliche Wirken eines Ambroise Paré, eines Felix Platter inspiriert. Dabei fühl-

${ }^{1}$ Herrn Dr. med. Charles Salzmann in Zürich möchte ich an dieser Stelle für mancherlei Anregung meinen Dank aussprechen.

2 Paul Diepgen, Geschichte der Medizin I, Berlin 1949, S. 276.

3 Heinrich Buess, Conrad Geßners Beziehungen zu Basel, Gesnerus 5 (1948) 1-29.

4 Bernhard Milt, Kurzbiographie von Conrad Geßner in Große Schweizer Forscher, Zürich 1939 , S. $50-52$. 
ten sich diese Männer viel weniger, als wir heute simplifizierend anzunehmen geneigt sind, im Gegensatz zur Vergangenheit; Paracelsus mit seiner ungestümen Ablehnung der gesamten antiken und arabischen Medizin wie des klaren Lateins als Umgangssprache der Gelehrten war in dieser Beziehung die große, von den besonneneren Geistern mißbilligte Ausnahme. Conrad Geßner und die allermeisten seiner Zeitgenossen achteten die alten Autoren als Männer von umfassenden Kenntnissen und reichem Wissen; hochfahrend an ihnen vorbeizugehen hatte man kein Recht, auch wenn man selber manche Dinge anders sah und zu neuen Schlüssen und Einsichten kam.

Ein so vielseitiger Mediziner und fruchtbarer Schriftsteller wie Geßner muß sich auch - wenigstens gelegentlich - zu Problemen des Sehens und über Augenleiden und deren Behandlung geäußert haben. Geßners Thesaurus medicinae practicae liegt freilich noch heute als Sammlung loser, handschriftlicher Notizen in der Zürcher Zentralbibliothek ${ }^{5}$ und wurde hier nicht berücksichtigt. Es genügt jedoch, Teile des gedruckten Werkes durchzusehen $^{6}$, um einen hinreichend deutlichen Eindruck von Geßners Ansichten in ophthalmologischen Dingen zu gewinnen.

Mittel zur Behandlung von Augenleiden führt Geßner im Thesaurus Evonymi Philiatri auf, in welchem er die Destillation in all ihren Modifikationen ausführlich darstellt, aber auch die Herstellung anderer Heilmittel - vorwiegend in flüssiger Form - beschreibt. Traditionsgemäß nehmen die Arzneien pflanzlicher Herkunft den breitesten Raum ein ${ }^{7}$. Sehr gewissenhaft nennt Geßner die Autoren, aus deren Schriften er geschöpft hat; gelegentlich läßt er diskret durchblicken, wenn er ihnen nicht ganz traut. So etwa

${ }^{5}$ MSS. 204 A-C. Vgl. über diese, 1596 von Geßners Schüler Caspar Wolf geordnete Sammlung Dieter Baumann, Psychiatrisches bei Conrad Geßner, Gesnerus 10 (1953) 123-150.

${ }^{6}$ Berücksichtigt wurden: a) Thesaurus Evonymi Philiatri, de remediis secretis liber physicus, medicus et partim etiam chymicus et oeconomicus, Zürich 1552, b) die Briefsammlungen, herausgegeben von CASPAR WoLf: Epistolarum medicinalium ... libri III, Zürich 1577, und von Johannes Hanhart: Epistolarum medicinalium ... liber quartus, Anhang zu Hanharts Biographie von Conrad Geßner, Winterthur 1824.

${ }^{7}$ Vgl. Bernhard Mitt, Chemisch-alchemistische Heilkunde und ihre Auswirkungen in Zürich, Vjschr. Naturforsch. Ges. Zürich 98 (1953) 179-215, über den «Thesaurus» besonders S. 192. 
bei der Beschreibung des Lebenswassers (aqua vitae) nach ArNald von VILLANOVA ${ }^{8}$ :

«Die am Haupt anzuwendenden Mittel sind viel wirksamer, wenn sie zu der Zeit aufgelegt werden, da der Mond im Widder steht usw. Wieweit ich dies für wahr halte, werde ich jetzt nicht sagen. Das aber will ich nicht verschweigen: je gelehrter in unserem Jahrhundert jemand ist, desto weniger zollt er derartigen Vorurteilen (persuasionibus) Tribut, welche die Araber in die Medizin hereingebracht haben.»

Die Tränke, deren Zubereitung Geßner zum allgemeinen Besten beschreibt, müssen oft wahre Panazeen gewesen sein. In der Vielfalt der ihnen zugeschriebenen Wirkungen erinnern sie durchaus an die volkstümlichen Wunderbalsame der Gegenwart, übertreffen diese ihre kläglichen Nachfahren aber freilich um ein Erkleckliches. So gibt es u.a. ein Kraft- oder Goldwasser (aqua virtutis sive aurea), zubereitet aus Salbei und anderen Kräutern, das nicht nur alle inneren Krankheiten, ferner Abszesse und Wunden heilt, sondern auch das Gesicht verjüngt, so daß ein Achtzigjähriger wie ein Dreißigjähriger aussieht. Zudem macht es den, der es trinkt, fröhlich, konserviert Wein und Gewürze, wehrt der Fäulnis, scheidet Quecksilber von Silber und ist bei mancherlei Erkrankungen bestimmter Körperteile von Nutzen: Es hilft bei Zahnweh, bei Krankheiten des Mundes (wenn man es über Nacht drin behalten kann!), bei Ozaena und Nasenkatarrh (wiederum, wenn man es in der Nase behält). Mit einem Federkiel ins Auge getropft, heilt es Entzündungen, Pusteln, Hornhauttrübungen und Pterygien. Reibt man damit den Kopf ein, so bringt es den grauen Star zum Verschwinden, der ja durch Herabrinnen krankhafter Säfte aus dem Hirn ins Auge erklärt wird: «emendat guttam ex catarrho cerebri nascentem.» Den uralten Irrtum über die Genese der Cataract hat erst Felix Platter mit anatomischen Argumenten widerlegt ${ }^{9}$.

Geßner kennt selbstverständlich auch besondere Augenwässer, die teils getrunken, teils lokal angewandt werden sollen. Die darin verarbeiteten Heilpflanzen finden sich im wesentlichen schon in der antiken Materia medica des Dioskorides aus dem 1. Jahrhundert n. Chr. aufgeführt ${ }^{10}$ : Rautenblatt (folium rutae) gegen Augenschmerzen, Salbei (salvia) gegen

8 Thesaurus, S. 114 ff.

${ }^{9}$ Felix Platter, Praxeos ... tractatus I. de functionum laesionibus, Basel 1602, cap. VII de visus laesione.

${ }^{10}$ s. Julius Hirschberg, Geschichte der Augenheilkunde, 1. Buch: Geschichte der Augenheilkunde im Altertum = 12. Band von Graefe/SAEmisch, Handbuch der gesamten Augenheilkunde, Leipzig 1899, Paragraph $125 \mathrm{ff}$. 
Hornhauterkrankungen, Fenchel (foeniculum) und Schöllkraut (Chelidonium) zur Stärkung der Sehkraft, Aloe für blutunterlaufene Lider, Myrrhe gegen Trübungen der Cornea, Wegwarte (sponsa solis) gegen Augenentzündungen. Auf diese Pflanze (cichorium L.) hält Geßner große Stücke: Das daraus zubereitete Heilwasser oder Öl «schärft die Sehkraft und heilt jegliche Augenkrankheit innert fünf Tagen »!11 Selbstverständlich fehlt unter den augenheilenden Pflanzen der Augentrost (euphragia ${ }^{12}$ ) nicht. Der Saft, der im Frühjahr nach dem Beschneiden der Reben aus den Schnittstellen tropft, lindert ebenfalls das Stechen und Brennen der Augen. Nicht nur gegen Entzündungen, sondern auch gegen den grauen Star (ad cataractam sive suffusionem) hilft ein Wasser, das neben den Extrakten verschiedener Pflanzen Kandiszucker, Zinkoxyd (tutia optima) und den so beliebten sanguis draconis enthält ${ }^{13}$. Diese wenigen Beispiele aus dem verwirrend reichhaltigen Arzneischatz Geßners mögen genügen. Geßner erscheint in seinem «Thesaurus» als gewissenhafter und geradezu begeisterter Sammler, der an jedem Fund - in der Literatur wie in der Natur - seine Freude hat und ihn mit Vergnügen seinen Zeitgenossen mitteilt. Mögen diese dann selbst erproben, was die Sache jeweils taugt!

\section{III}

Etwas ophthalmologische Kasuistik enthält das dritte Buch der WoLFschen Briefsammlung. Über drei Vierteljahre hin erstreckt sich die Korrespondenz mit Johannes Kosmas Holzach, dem aus angesehener Basler Familie stammenden Schaffhauser Physicus, über das Augenleiden seiner Frau. Da wir Holzachs Briefe nicht kennen, müssen wir die Natur des offensichtlich hartnäckigen Übels aus Geßners Antworten erschließen. Der große Gelehrte beginnt sein Consilium mit einer Entschuldigung ${ }^{14}$ :

«Augenkrankheiten zu kurieren bin ich nicht gewohnt; denn zum einen sehe ich selber sehr schlecht (hebetissimo visu sum) und könnte manche Augenleiden nicht gut unterscheiden; zum andern möchte ich unseren Kurpfuschern (empirici) und Augenpraktikern

11 Thesaurus, S. 259 f.

12 Über die Geschichte der Euphrasia s. Marianne Schorr, Klin. Mbl. Augenheilkde. 131 (1957) 834-840.

13 Thesaurus, S. 259.

${ }^{14}$ Brief an Holzach vom 15. November 1560, Epistolarum fol. 84 recto. 
(ophthalmici) auch etwas übrig lassen. Unser Steinschneider Meister Peter ${ }^{15} \ldots$ ist in der Augenbehandlung wohl erfahren; wessen Augen aber jener BumanN (oder Baumann: «Bumannus», zürichdeutsch «Buume») bisher geheilt hat - dieser waghalsige Alles-

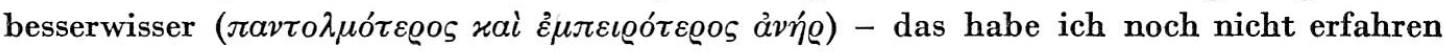
Man sagt, er wage sich sogar an den Grauen Star.»

Dann kommt Geßner zu seinem Therapievorschlag:

«Ich selber habe bei einer seit langem bestehenden Augenentzündung früher einmal mit gutem Erfolg die Venen unter der Zunge schneiden lassen. Wenn Du das bei Deiner Frau tun willst, so purgiere gleichzeitig ihren Körper und setze sie auf Diät. Dann kannst Du für sie ein Wasser etwa auf folgende Art destillieren ...»

Das Heilwasser enthält neben mancherlei pflanzlichen Ingredienzen Hirschhorn, Eisenpulver und Eisenfeilspäne; Eisen und Kupfer werden ebenfalls schon von Dioskorides als Adstringentien empfohlen ${ }^{16}$. Der Destillationsprozeß wird mehrere Tage dauern. Geßner fährt fort:

«Diese Flüssigkeit habe ich nie zubereitet oder zubereiten sehen, aber ohne Zweifel wird sie ganz vorzüglich sein.»

Bis sie bereit ist, kann Zinksalbe (collyrium Nihili) ${ }^{17}$ auf die Lider und Augenwinkel aufgetragen werden, oder aber - als stärkeres Adstringens Kupfervitriol (chalcanthum).

«Zuerst aber sollen die Augen mit Honigwein, in dem man ein bißchen Bockshornklee ${ }^{18}$ gekocht hat, gewaschen werden ...»

Schließlich ermahnt Geßner seinen Freund, sich zu vergewissern, daß nicht eine Tränenfistel vorliegt oder Encanthis ${ }^{19}$, eine Wucherung der Caruncula lacrimalis, welche nach antiker Auffassung zu Tränenfluß und dergleichen hätte Anlaß geben können.

Der «liquor procul dubio optimus» - sofern er zubereitet wurde - hat die in ihn gesetzten Hoffnungen leider nicht erfüllt. Geßner muß im folgen-

15 Petrus Figulus = Peter Hafner, 1526-1592, weitbekannter Bruch- und Steinoperateur (freundliche Mitteilung von Herrn Dr. Salzmans).

${ }^{16}$ Hirschberg, a.a. O., Paragraphen 138, 140.

17 Nihilum album, nix alba, lana philosophica, tutia alba, pompholyx: Zink-Oxyd. Colly-

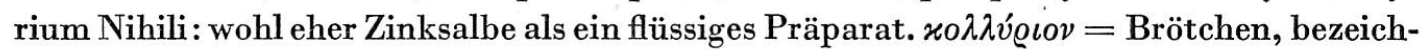
nete ursprünglich eine feste Form von Medikamentenzubereitung, s. HirschBerg, a. a. O., Paragraphen 144-146.

${ }^{18}$ Foenograecum, jetzt Trigonella foeni-graeci: «Was heute Kamillentee, war Bockshornklee-Abkochung bei den Alten», Hirschberg, a.a. O., Paragraph 127, S. 212, Fußnote 1.

19 s. Hirschberg. a. a. O., Paragraph 240, nach Paulos von Aigina. 
den Sommer von seinem eigenen Zinkpulver nach Schaffhausen schicken, da Dr. Holzach - oder seine augenleidende Frau? - sich nicht davon überzeugen läßt, daß der Pulvis Nihili des Schaffhauser Apothekers genau so gut sein soll wie die Tutia alba, die der große Conrad Geßner besitzt. Dieser schließt die Korrespondenz über den langwierigen Fall wie folgt ${ }^{20}$ :

«Daß ich selber jene Knötchen an den Augen je gesehen hätte, erinnere ich mich nicht; ich bin ja ein kurzsichtiger Mann (homo lusciosus), der Augenleiden nicht gern behandelt, damit man mir nicht mein eigenes Übel vorhalte. Meister Peter sagt, er habe solche Knötchen gesehen, aber vereitert. Vielleicht befindet sich Deine Frau seither schon besser, was wir hoffen - wenn nicht, so wirst Du dank Deiner Bildung und Umsicht aufs beste für sie sorgen.»

Wir können aus den mitgeteilten Briefstellen auf eine chronische BlepharoConjunctivitis mit multiplen und wohl rezidivierenden Chalazien schließen. Das an sich ungefährliche Leiden kann die Patienten sehr plagen und ist auch heute gelegentlich eine wahre crux medici. Vielleicht sollten wir - da es sich um eine seborrhoische Erscheinung handeln kann - wieder mehr daran denken, unsere Patienten auf Diät zu setzen!

In Übereinstimmung mit der antiken Humoralpathologie sieht Conrad Geßner in den Augenleiden weniger lokale Krankheiten eigener Art als vielmehr Manifestaiionen einer allgemeinen Störung im Organismus. Die Eiterung des Tränensackes, den Aigilops, behandelt er mit Aderlaß aus den sublingualen Venen und Purgantien, also rein humoral ${ }^{21}$.

\section{IV}

Eigentliche Sehstörungen sind es gewesen, die den Danziger Arzt Johannes Placotomus ${ }^{22}$ bewogen haben, bei Geßner Rat zu suchen. Dem vielseitigen, von Melanchthon hochgeschätzten Manne mußte wohl der im fernen Zürich wirkende Gelehrte nicht nur wegen der gemeinsamen wissenschaftlichen Interessen, sondern auch wegen seiner ausgesprochenen, evangelischen Überzeugung besonders vertrauenswürdig erscheinen. In dem

${ }^{20}$ Brief an Holzach vom 18. Juli 1561, Epist. fol. 85 recto.

${ }^{21}$ Brief an Holzach vom 31. Januar 1562, Epist. fol. 85 verso.

22 Johannes Placotomus = Brettschneider, 1514-1576/77, Dr. med. zu Wittenberg 1540/41, Professor in Königsberg, ab 1552 Stadtsyndicus und Verwalter der Ratsapotheke in Danzig, publizierte 1553 über chemische Destillationen und nahm sich um die Organisation des höheren Schulwesens an (Allgemeine deutsche Biographie). 
einzigen Brief an Placotomus, den die Wolfsche Sammlung enthält ${ }^{23}$, geht Geßner zunächst auf die wahrscheinliche Kurzsichtigkeit ein, die den gelehrten Freund plagt:

* Offensichtlich verlieren sich die Sehgeister zu schnell (spiritus visivos dissipari apparet), da Du ferne Dinge nicht erkennen kannst, sondern nur die nächstgelegenen. Mir geht es seit jungen Jahren (ab adolescentia) ebenso: ich bin nämlich schwachsichtig und myop (lusciosus et $\left.\mu v \omega^{\prime} \varphi\right)$; um in die Ferne ein bißchen etwas zu sehen, benutze ich daher eine Art Brille (conspiciliorum genere utor), welche die Spiritus sammelt und die Dinge kleiner erscheinen läßt. Ich möchte gerne wissen, ob Du Dir mit einer Brille behilfst und mit welcher Art. Ich glaube tatsächlich, daß auch Du kurzsichtig (myops) bist. Dieses Übel ist unheilbar, hat aber das eine Gute, daß mit dem Alter die Augen nicht schlechter werden. Bei diesem Leiden kommt es nämlich nicht zur Eindickung der Spiritus, die sonst bei den alten Leuten zur Sehschwäche führt, denn die Myopiasis selbst rührt von einer besonderen Feinheit des Spiritus her: dies ist der Grund, weshalb dieser sich so leicht verliert.»

Zur Erklärung des Seh-Vorganges hält sich also Geßner an die antike Fühlfaden-, Sehstrahlen- oder Emanationstheorie, die nach PolyaK wie nach Hummel auf die Schule des Pythagoras zurückgeht, und die sich ausgezeichnet mit der Pneuma-Lehre Galens vertrug: Aus dem Auge treten Strahlen oder eben die Spiritus visivi aus und stellen - Fühlern gleich - die Verbindung zwischen Sinnesorgan und Objekt, zwischen Auge und sichtbarer Umwelt, her. Diese Theorie galt freilich nicht unangefochten: Schon Aristoteles hatte eine Wirkung des Objektes auf das Auge (und nicht umgekehrt) postuliert, und der Araber IbN al-Haitham (ALHAZEN, um $1000 \mathrm{n}$. Chr.) hatte die Lehre, daß beim Sehen irgend etwas aus dem Auge herauskomme, klar als Irrtum erkannt ${ }^{24}$. Aber die falsche Theorie hielt sich trotzdem hartnäckig, denn sie paßte in das Gesamtbild, das man sich vom Organismus machte, und erlaubte eine offenbar befriedigende Deutung vieler Sehstörungen: Warum sieht der Kurzsichtige nicht gut in die Ferne, wohl aber in die Nähe? - Weil eben die allzu feinen Spiritus zerstieben, lange bevor sie entfernte Gegenstände erreichen! Es blieb Johannes KePler vorbehalten, zu Beginn des 17.Jahrhunderts die Refraktionsanomalien richtig zu deuten.

${ }^{23}$ Brief Gessners an Placotomus (Placetomus) vom 27. März 1557, Epist. fol. 136 verso bis 137 verso.

${ }^{24} \mathrm{Zu}$ den Sehtheorien der Griechen und Araber vgl. a) Stephen Polyak, The Vertebrate Visual System, Chicago 1957, S. 11/12 und 17 ff.; b) Walter Hummer, Die Physiologie des Auges in der arabischen Medizin, Diss. Münster in Westfalen 1949 (maschinengeschrieben); c) Matthias Schramm, Zur Entwicklung der physiologischen Optik in der arabischen Literatur, Sudhoffs Arch. Gesch. Med. 43 (1959) 289-316. 
Placotomus leidet zudem noch an Doppeltsehen. Obwohl Aristoteles, Alexander von Aphrodisias und Cassius sich in ihren «Problemen» mit dem Doppeltsehen der Betrunkenen abgegeben haben, kann Geßner Natur und Ursache der Störung nicht zu seiner Befriedigung erklären. Einig scheinen sich die genannten Autoritäten darüber zu sein, daß eine asymmetrische Lage der Augen (inaequalitas situs oculorum) vorliege.

«Wenn man mit dem Finger auf ein Auge drückt, so daß es höher als das andere steht, verdoppeln sich die betrachteten Objekte. ${ }^{25}$

Anders als die Kurzsichtigkeit kann man die Diplopie nicht einfach durch abnorme Zerstreuung der Spiritus erklären, vielmehr muß deren kontinuierlicher Fluß von den Augen zum Objekt gestört sein, und zwar so, daß die Spiritus aus den beiden Augen nicht gleichzeitig beim Objekt anlangen. Dies kann die Folge der Verlagerung eines Auges, eines Sehnerven oder eines der Humores im Innern des Auges sein. Es ist aber auch möglich, daß die Vereinigung der beiden Sehnerven (nervorum opticorum coniunctio) durch eine pathogene Flüssigkeit oder sonst einen störenden Körper unterbrochen ist. Placotomus möge die Sache weiter überdenken:

«Vielleicht kannst Du die Ursache herausfinden - dann bestünde einige Hoffnung auf Heilung. Andernfalls wirst Du Dich in Gottes Willen fügen!»

Auf das Gesagte zurückblickend, können wir uns kurz fassen mit Bezug auf Geßners ophthalmologische Konzeptionen: Sie sind rein galenisch. Dies gilt für die Physiologie des Gesichtssinnes und ihre Störungen, die sich Geßner mit Hilfe der Spiritus visivi, der Sehgeister, erklärt, welche vom Hirn in die Augen und von den Augen zu den betrachteten Dingen strömen, und es gilt für die Pathologie der Augenkrankheiten, die als Ausdruck humoraler Störungen im Organismus aufgefaßt und entsprechend behandelt werden. Die ableitenden Maßnahmen, die Geßner - soweit unsere Untersuchung reicht empfohlen hat, zeichnen sich durch eine humane Milde aus: Wohl wird kräftig purgiert und zur Ader gelassen; aber es ist nicht die Rede von künstlichen, mit dem Glüheisen gesetzten Geschwüren oder von den Haarseilen im Nacken, auf die Geßners Zeitgenosse Ambroise Paré so große

25 «Durch Verschieben eines Bulbus entstehen Doppelbilder.» So faßt K. E. RothschUH GaLens Auffassung zusammen: Geschichte der Physiologie, Berlin/Göttingen/Heidelberg 1953, S. 17. 
Stücke hielt ${ }^{26}$. Die Lokalbehandlung mit Einträufeln heilkräftiger Flüssigkeiten, Abwaschungen, Salbenanwendung hat in der Augenbehandlung seit jeher ihre Holle gespielt und spielt sie auch bei Conrad Geßner; der augenärztliche Medikamentenschatz ist im wesentlichen derjenige der Antike.

Nicht ohne Interesse sind die Aufschlüsse, die wir aus den zitierten Briefen über Geßners Einstellung zur Augenheilkunde und zu seiner eigenen Kurzsichtigkeit gewinnen konnten. Für die Optik hat er sich offensichtlich nicht interessiert: Schon damals konnte selbst der universellste Gelehrte nicht alle Bereiche menschlichen Wissens kultivieren. Mit der Behandlung der Augenleiden mochte er sich nach seinen eigenen Worten nicht gern abgeben: Selbst mit einem solchen Übel - der Kurzsichtigkeit - behaftet, fühlte er sich dazu nicht kompetent. Die Kurzsichtigkeit ist damals im täglichen Leben bestimmt weit hinderlicher gewesen als jetzt: Die Brille, seit dem Ende des 13. Jahrhunderts in Gebrauch, war ein wertvoller Notbehelf; aber weder optisch noch kosmetisch hatte sie auch nur annähernd den Grad von Vollkommenheit erreicht, der sie heute zur unentbehrlichen und gern getragenen Ergänzung so manches Gesichtes macht. Der kurzsichtige Arzt des 16. Jahrhunderts war also tatsächlich behindert gegenüber den Patienten, die es bekanntlich schätzen, wenn man ihnen gleich ansieht, weshalb sie zu uns kommen. Aber überhaupt war Geßner ganz allgemein weit mehr Gelehrter und Humanist als ärztlicher Praktiker :

«Beschränkt und dürftig ist meine Praxis. Wir sind hier sehr viele (permulti - !) Ärzte; ich ziehe mich auf meine Studien zurück und suche die Praxis nicht. ${ }^{27}$

Ohnehin waren es meist die Chirurgen, welche die Augenkranken behandelten, neben den ausgebildeteten und tüchtigen Wundärzten und Starstechern leider auch Quacksalber und von Markt zu Markt ziehende Wunderdoktoren.

Conrad Geßner hat die guten Seiten der Myopie ebenfalls zu schätzen gewußt. Sie hat es ihm erlaubt, auch in späteren Jahren ohne Schwierigkeiten unermüdlich weiter zu lesen und zu schreiben, und sie hat es ihm ermöglicht, den Bau seiner geliebten Pflanzen in den feinsten Einzelheiten besonders exakt zu erkennen und wiederzugeben.

${ }^{26}$ Vgl. M. H. Koelbing, Bull. Mém. Soc. Franç. Opht. 73 (1960) 675-680.

${ }^{27}$ Brief an Holzach vom 2. April (1564 ?), epist. fol. 87 recto. 